\title{
A Case Report Illustrating the Spontaneous Vision Recovery After Inadvertent Central Descemetorhexis During Anterior Capsulotomy
}

This article was published in the following Dove Press journal: International Medical Case Reports Journal

\author{
Drahomíra Barákovál,2 \\ Denisa Darsová \\ 'Gemini Eye Clinic a.s., Prague, Czech \\ Republic; ${ }^{2}$ Department of \\ Ophthalmology, Second Faculty of \\ Medicine, Charles University Prague and \\ Motol University Hospital, Prague, Czech \\ Republic
}

\begin{abstract}
Purpose: To demonstrate an unusual mechanism of iatrogenic, central descemetorhexis (DMR) during cataract surgery and subsequent rare spontaneous visual acuity improvement within 2 months after inadvertent surgical complication.

Patients and Methods: A 81 year old woman underwent cataract surgery complicated by the loss of a $4.8 \times 4.75 \mathrm{~mm}$ diameter central area of Descemet membrane. Perioperative video recording documented the DMR formation during continuous curvilinear capsulorhexis creation.

Results: Postoperatively, severe corneal edema with folds in the remaining Descemet membrane were observed. The patient was managed conservatively. The corneal edema gradually resolved over 2 months with improving of visual acuity from counting fingers to 20/20.

Conclusion: Unlike Descemet membrane detachment, descemetorhexis is a rare complication after intraocular surgery. The case report identifies a previously unknown mechanism of DMR formation during anterior capsulotomy creation. Loss of Descemet membrane may be managed conservatively in an otherwise healthy cornea with good final visual outcome without the need for surgical intervention.
\end{abstract}

Keywords: cataract surgery, corneal edema, Descemet membrane detachment, descemetorhexis, loss of Descemet membrane, spontaneous vision recovery

\section{Introduction}

Descemetorhexis describes complete removal of Descemet membrane leading to a potentially sight-threatening condition. Analogous to a rhegmatogenous retinal detachment, descemetorhexis may occur as a rare complication of trauma, corneal ectasia, or during cataract surgery when there is a tear in the Descemet membrane. The fluid from the anterior chamber may than accumulate between the membrane and the overlaying stroma leading to a subsequent partial or complete ablation (Descemet membrane detachment) or complete separation (Descemetorhexis) of the membrane. If left untreated, resulting corneal edema may persist, leading to corneal decompensation and visual loss. The conventional surgical treatment strategies include Descemet membrane endothelial keratoplasty, Descemet stripping (automated) endothelial keratoplasty, pre-Descemet's endothelial keratoplasty, femtosecond laser-assisted keratoplasty, or penetrating keratoplasty to repair the missing Descemet membrane. ${ }^{1-3}$ To avoid complications of long-term immunosuppressive treatment after keratoplasty, new salvage agents are the subject of intense research. ${ }^{4}$
Correspondence: Denisa Darsová Gemini Eye Clinic a.s., U Kř́žku 572 , Prague, Průhonice, 252 43, Czech Republic

Email denisa.darsova@gmail.com
International Medical Case Reports Journal 202I:I4 45-49 
In our case report, we have documented a previously undescribed mechanism of descemetorhexis during anterior capsulotomy creation. We report an unusual case of full vision recovery in a patient with large iatrogenic descemetorhexis including persistent central corneal edema. After 2 months of conservative treatment, edema underwent spontaneous resolution.

\section{Patients and Methods}

Written informed consent has been provided by the patient to have the case report details and any accompanying images published prior to publication. Institutional approval was not required to publish the case details. An 81 year old woman presented with immature senile cataract for phacoemulsification in our centre. Best corrected visual acuity was 0.05 (Snellen chart 6-m distance, high contrast). Slit lamp examination was remarkable for a clear cornea with normal appearing endothelium. This was confirmed by specular endothelial microscopy and cell count that showed no significant difference between the two eyes before surgery $\left(2,455\right.$ cells $/ \mathrm{mm}^{2}$ on the right eye and $2,449 \mathrm{cells} / \mathrm{mm}^{2}$ on the left eye, Nidek CEM-530; Nidek Co., Ltd, Japan). Pentacam pachymetry (Oculus Pentacam 70,700, Oculus, Germany) revealed central corneal thickness of $590 \mu \mathrm{m}$ in the right eye and $618 \mu \mathrm{m}$ in the left eye. Otherwise, there were no pathological findings on the anterior or posterior segment of both eyes. The ocular history was characteristic of mild hypermetropia and blepharoplasty. The eye medical history was unremarkable.

Written informed consent and the EU General Data Protection Regulation Form were obtained by the patient prior to the operation. Cataract extraction was performed in a standard way via temporal clear corneal incision placed at 180 degrees under topical anesthesia. A small side incision was made with a number $15 \mathrm{knife}$. Intracameral anesthetics and a disperse ocular viscoelastic device (OVD) were inserted through the main incision. The operation continued with the creation of a continuous curvilinear capsulotomy. Following the capsule extraction, the surgeon noted that there was a defect in the central part of the Descemet membrane beside the central circular border after the CCC which dropped to the main clear corneal incision (Figure 1C).

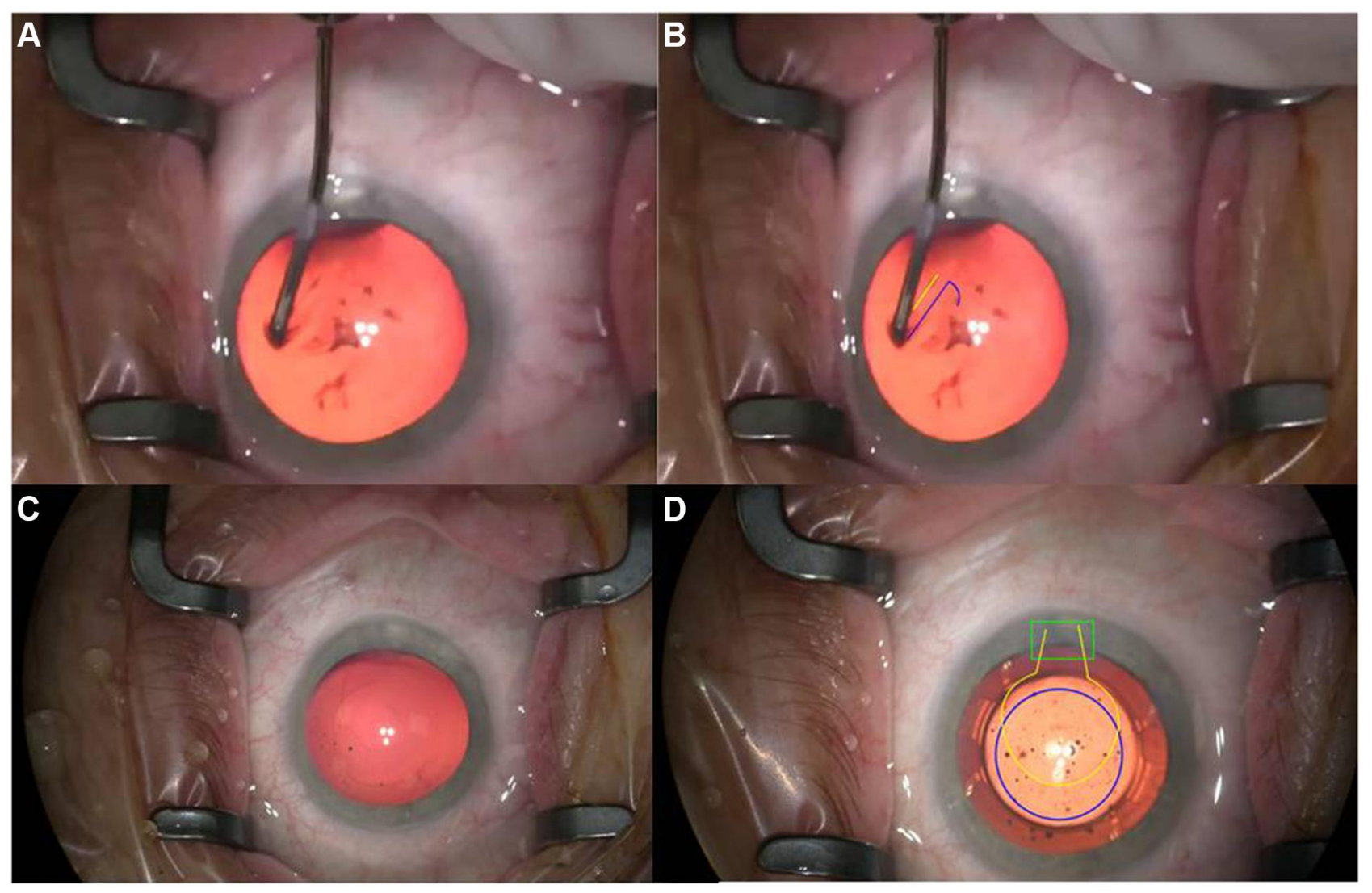

Figure I (A) Native picture of continuous curvilinear circular capsulotomy with Descemet's membrane; (B) preparation of both Descemet's membrane (yellow line) and anterior capsule (purple line) during continuous curvilinear circular capsulotomy; (C) native picture after creation of complete capsulotomy; (D) microscopic photo after creation of complete capsulotomy. Purple line marks continuous curvilinear capsulotomy, yellow line marks descemetorhexis and green line clear corneal incision. 
Phacoemulsification and intracapsular lens implantation were completed without complications. At the end of the operation, a clear cornea with central defect in Descemet membrane was present (Figure 1C and D).

Conservative management in the form of dexamethasone (Dexamethasone WZW Polfa $1 \mathrm{mg} / \mathrm{mL}$, Warszawskie Zaklady Farmaceutyczne Polfa S.A., Poland), antibiotic eye drops (Tobradex $3 \mathrm{mg} / \mathrm{mL}+1 \mathrm{mg} / \mathrm{mL}$, Novartis s.r.o., Czech Republic), antiglaucomatic (Timolol-POS 0.5\% $5 \mathrm{mg} / \mathrm{mL}$, Ursapharm, Czech Republic) and non-steroidal antiinflammatory eye drops (Yellox $0.9 \mathrm{mg} / \mathrm{mL}$, Pharma Swiss s.r.o., Czech Republic) were started.

\section{Results}

On the first postoperative day, best CDVA was 20/400 in operated eye (Snellen chart 6-m distance). Slit lamp examination revealed central corneal stromal edema with folds in the remaining Descemet's membrane (Figure 2A). The size of the descemetorhexis, documented on perioperative microscopic photography (Figure 1C and D), was measured as $4.88 \times 4.75 \mathrm{~mm}$ and the continuous curvilinear capsulotomy (CCC) dimension $5.28 \times 5.38 \mathrm{~mm}$ against the corneal horizontal diameter (white-to-white) $11.6 \mathrm{~mm}$. After 7 days, best CDVA was reduced to counting the fingers. Central corneal edema progressed as the intraocular lens was not clearly visible (Figure 2B). However, the cornea become clearer for 1 month with continued conservative treatment without antiglaucomatics. Optical coherence tomography (OCT, RTVue-100, version 6.9, Optovue Inc., USA) recorded a large oval $480 \mu \mathrm{m}$ central descemetorhexis with folding of the edge of the membrane in the anterior chamber. Best CDVA improved to $20 / 80$ and 2 months after cataract surgery was 20/20. Corneal edema completely resolved (Figure $2 \mathrm{C}$ and $\mathrm{D}$ ). In the OCT scan 13 months after surgery, the Descemet membrane line was hyperechogenic (Figure 3). Specular endothelial microscopy showed a decrease in the number of the endothelial cells $\left(1,087\right.$ cells $\left./ \mathrm{mm}^{2}\right)$. The percentage of hexagonal cells (polymorphism) was 53\% and the index of the extent of variation (polymegathism) was $52 \%$.

\section{Discussion}

Surgically induced descemetorhexis is a rare but wellknown complication of surgical trauma from instruments, OVD, and intraocular lenses at the incision site. Most cases were created during phacoemulsification with clear corneal incisions, ${ }^{1,2,5}$ one case during scleral tunnel preparation with a crescent knife and keratome, ${ }^{4}$ and one

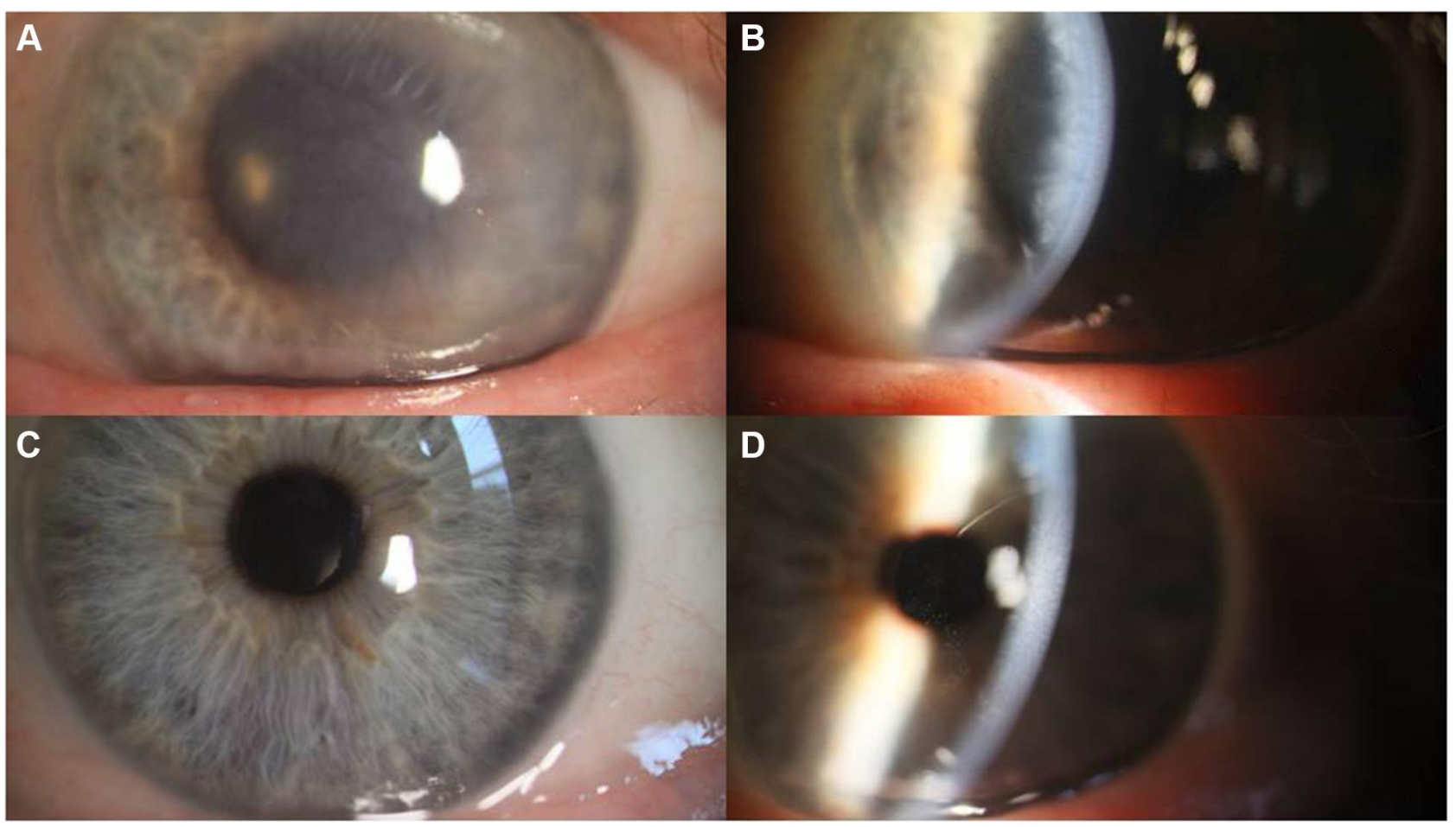

Figure 2 (A) Microscopic finding of corneal stromal edema on the first day after cataract surgery; (B) progression of stromal corneal edema 7 days after cataract surgery; (C) microscopic picture of the cornea 2 months after cataract surgery; (D) slit lamp picture of the cornea 2 months after cataract surgery. 


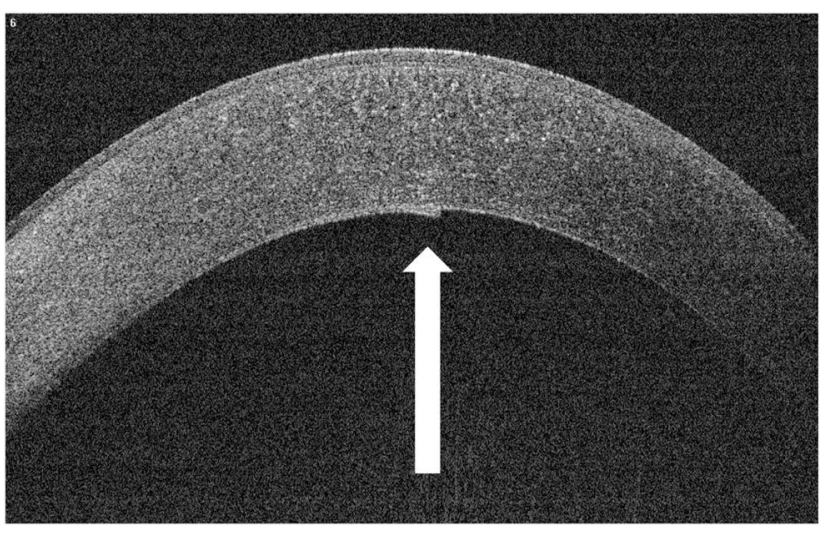

Figure 3 Hyperechogenic Descemet membrane line (arrow) on the OCT 13 months after surgery.

during intraocular injection of OVD. ${ }^{6}$ In our case, the tear of Descemet membrane probably occurred during clear corneal incision. Subsequently, the OVD was inadvertently partially injected intracorneally, separating Descemet membrane from the stroma and flattening it onto the anterior capsule. Following the creation of continuous curvilinear capsulotomy, the surgeon unconsciously prepared both the Descemet's membrane and anterior capsule (Figure 1A and B). The surgery was completed without extending the detachment. Loss of Descemet's membrane leads to a sharp decrease of visual acuity due to corneal edema, as was seen in our case (Figure 2A and B). In view of the normal corneal findings on the unoperated eye, suggesting good postoperative recovery on the operated eye, we decided for conservative treatment. However, it is accepted that prolonged observation of corneal edema carries a risk of bullous keratopathy, corneal fibrosis, and scarring and microbial keratitis. ${ }^{2}$ Nevertheless in our case of descemetorhexis, a 2 month central corneal edema has not surprisingly lead to these complications.

It is assumed that the corneal endothelium of adult humans has a poor regenerative capacity. ${ }^{1,2}$ This assumption is confirmed by the decrease in endothelial cell density with age. However, several studies have proved that cell proliferation and migration from the surrounding endothelial wound edge and peripheral endothelial cells can spontaneously heal the Descemet membrane deficit. ${ }^{7-9}$ The paracrine effect of growth factors secreted by the remaining endothelial cells can enhance this migration. The smooth surface of a thin new basement membrane can extend between the two parts of Descemet membrane along the stromal wound surface, enabling endothelial cell migration and redistribution. Increase in size of the remaining endothelial cells helps to reform the endothelial cell layer. ${ }^{10}$ Specular microscopy performed 13 months after the surgery supports this concept of endothelial cell healing activity with an increased index of polymorphism and polymegathism. Increased reflectivity in the area of Descemet membrane on the OCT was seen, probably due to fibrosis and scarring.

Corneas with preexisting endothelial dysfunction, abnormalities in the attachment between the membrane and the overlaying stroma, and anomalous anatomy of the membrane show a slower return to preoperative pachymetry and good visual acuity. ${ }^{7,8}$ Recent studies have shown promising results after Rho-associated kinase inhibitors treatment as a support for endothelial healing in cases of central descemetorhexis. ${ }^{4} \mathrm{We}$ experienced a slow spontaneous improvement in corneal transparency 7 days after the surgery and after 2 months, descemetorhexis in $4.88 \times 4.75 \mathrm{~mm}$ diameter completely healed with CDVA 20/20 without requiring surgical management. Thus, endothelial cells restored their function.

\section{Conclusion}

In the case of a normal corneal finding on the unoperated eye and when the corneal edema appears to be showing a gradual resolution within 3 months, loss of more than $4 \mathrm{~mm}$ large central area of Descemet's membrane from cataract surgery may be managed conservatively with good final visual outcome. Descemetorhexis may unusually occur during anterior capsulotomy creation.

\section{Author Contributions}

All authors contributed to the data analysis, drafting, or revising of the article, have agreed on the journal to which the article will be submitted, gave final approval of the version to be published, and agree to be accountable for all aspects of the work.

\section{Disclosure}

The authors report no conflicts of interest in this work.

\section{References}

1. Braunstein RE, Airiani S, Chang MA, Odrich MG. Corneal edema resolution after "Descemetorhexis". J Cataract Refract Surg. 2003;29:1436-1439.

2. Samarawickrama C, Beltz J, Chan E. Descemet's membrane detachments post cataract surgery: a management paradigm. Int J Ophthalmol. 2016;9:1839-1842.

3. Chich A, Lugo M, Kowing D. Descemet stripping and automated endothelial keratoplasty: an alternative to penetrating keratoplasty. Optom Vis Sci. 2008;85:152-157. 
4. Moloney G, Kerdraon Y, Beheregaray S. Descemetorhexis without rafting for Fuchs endothelial dystrophy-supplementation with topical ripasudil. Cornea. 2017;36:642-648.

5. Aggarval A, Jacob S, Aggarval S. Iatrogenic descemetorhexis as a complication of phacoemulsification. J Cataract Refract Surg. 2006;32:895-897.

6. Srivastava VK, Singh A, Chowdhary R. Spontaneous resolution of corneal oedema after inadvertent descemetorhexis during cataract surgery. Med J Armed Forces India. 2010;66:177-179.

7. Pan JCH, Eong A. Spontaneous resolution of corneal oedema after inadvertent „Descemethorhexis“ during cataract surgery. Clin Exp Ophthalmol. 2006;34::896-897.
8. Stewart RMK, Hiscott PS, Kaye SB. Endothelial migration and new descemet membrane after endothelial keratoplasty. Am J Ophthalmol. 2009;149:521-527.

9. Ruhe K, Finis D, Fuchsluger T, Geerling G. Spontane Visuserholung durch Endothelzellmigration bei Großem, Zentralem Postoperativem Descemetdefekt. Klin Monatsbl Augenheilkd. 2012;229:632-634.

10. Van den Bogerd B, Dhubhghaill SN, Koppen C, et al. A review of the evidence for in vivo corneal endothelial regeneration. Surv Ophthalmol. 2018;63:149-165.

\section{Publish your work in this journal}

The International Medical Case Reports Journal is an international, peer-reviewed open-access journal publishing original case reports from all medical specialties. Previously unpublished medical posters are also accepted relating to any area of clinical or preclinica science. Submissions should not normally exceed 2,000 words or 4 published pages including figures, diagrams and references. The manuscript management system is completely online and includes a very quick and fair peer-review system, which is all easy to use. Visit http://www.dovepress.com/testimonials.php to read real quotes from published authors. 